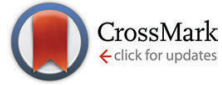

Cite this: Phys. Chem. Chem. Phys., 2015, 17, 17471

\title{
Exceptional adsorption-induced cluster and network deformation in the flexible metal-organic framework DUT-8(Ni) observed by in situ X-ray diffraction and EXAFS $\uparrow$
}

\author{
Volodymyr Bon, ${ }^{a}$ Nicole Klein, $\ddagger^{a}$ Irena Senkovska, ${ }^{a}$ Andreas Heerwig, $\S^{a}$

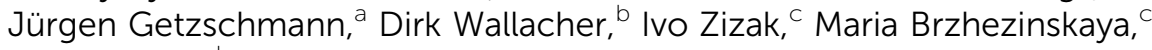 \\ Uwe Mueller ${ }^{d}$ and Stefan Kaskel*a
}

\begin{abstract}
The "gate opening" mechanism in the highly flexible MOF $\mathrm{Ni}_{2}(2,6-\mathrm{ndc})_{2}$ dabco (DUT-8(Ni), DUT = Dresden University of Technology) with unprecedented unit cell volume change was elucidated in detail using combined single crystal X-ray diffraction, in situ XRD and EXAFS techniques. The analysis of the crystal structures of closed pore (cp) and large pore (Ip) phases reveals a drastic and unique unit cell volume expansion of up to $254 \%$, caused by adsorption of gases, surpassing other gas-pressure switchable MOFs significantly. To a certain extent, the structural deformation is specific for the guest molecule triggering the transformation due to subtle differences in adsorption enthalpy, shape, and kinetic diameter of the guest. Combined adsorption and powder diffraction experiments using nitrogen (77 K), carbon dioxide $(195 \mathrm{~K})$, and $n$-butane $(272.5 \mathrm{~K})$ as a probe molecules reveal a one-step structural transformation from $\mathbf{c p}$ to Ip. In contrast, adsorption of ethane $(185 \mathrm{~K})$ or ethylene $(169 \mathrm{~K})$ results in a two-step transformation with the formation of intermediate phases. In situ EXAFS during nitrogen adsorption was used for the first time to monitor the local coordination geometry of the metal atoms during the structural transformation in flexible MOFs revealing a unique local deformation of the nickel-based paddle-wheel node.
\end{abstract}

Received 14th April 2015, Accepted 9th June 2015

DOI: $10.1039 / c 5 c p 02180 d$

www.rsc.org/pccp other porous crystalline materials is reversible structural switchability between non porous (closed pore structure) and porous (open pore structure) polymorphs. ${ }^{7}$ This novel (also termed 3rd) generation of coordination polymers ${ }^{8}$ is capable to perform a step-wise structural transformation as a response to external stimuli, such as guest molecules, electromagnetic radiation, temperature or pressure. Although more than 20.000 MOFs are known today only less than 100 of such switchable 3rd generation MOFs are structurally characterized and the theoretical elucidation of flexibility phenomenon is still in its infancy. Nevertheless, such MOFs already find a wide interest as switchable or highly selective gas adsorbents, ${ }^{9-12}$ threshold sensors, ${ }^{13,14}$ stimuli responsive drug delivery agents, ${ }^{15}$ switchable catalysts ${ }^{16}$ etc.

In order to establish a rational design, a detailed understanding of linker-cluster hinge deformation energies and atom rearrangement in the cluster nodes responsible for a ferroelastic deformation of the framework during the switching process is essential, as it represents a prerequisite for the effective prediction and recognition of the potential application fields as well as for understanding of driving forces, may it be derived from internal (adsorption induced) or external pressure. For such an understanding it is essential to elucidate crystal structures forming during the transformation induced by gas adsorption. 
However, the monitoring of the structural evolution during the "gate opening" in situ is challenging. So far only few groups have realized the parallelized collection of diffraction and adsorption data. ${ }^{17-21}$ In general, single crystal data during adsorption are very rare and sophisticated and thus powder diffraction is used giving good insight into structural framework deformation. ${ }^{22-24}$ For example, breathable MIL-53 family materials were successfully studied by in situ adsorption. ${ }^{21,25-27}$ However, the visualization of small local changes from Rietveld refinement even in combination with simulation is sometimes ambiguous. In this context the parallelized use of local probes is essential to confirm important structural details.

In the following we will for the first time show how a combination of single crystal and powder XRD with EXAFS elucidates precisely the structural changes of a highly flexible MOF, DUT$8(\mathrm{Ni})$, showing unprecedented unit cell volume expansion of up to $254 \%$ upon "gate opening". Not only the structures of the terminal states of the compounds (so called large pore (lp) phase and the closed pore (cp) phase) could by visualized on the atomistic level, but also the gas filled structural phases of the material were solved from in situ data collected during the adsorption of gases. We have focused especially on gases being important in the fields of energy storage, separation and environmental applications, namely carbon dioxide (195 K), ethane (185 K), ethylene (169 K), $n$-butane (273 K), and nitrogen (77 K). The measurements reveal essential mechanistic insights and may have a high impact for the development of 3rd generation MOFs for technical applications.

\section{Results and discussions}

DUT-8(Ni) is unique and anomalous among the pillar-layered compounds because of the huge pore volume expansion unattained in other pillared layer systems. ${ }^{28}$ The layers constructed of Ni paddle-wheels interconnected by 2,6-ndc linkers are pillared by dabco molecules, yielding a 3D framework with the $\alpha$-Po topology. The as-made compound crystallizes in $P 4 / n$ space group with 2 formula units per unit cell and contains square channels with limiting pore diameter of $9.0 \AA$ (Fig. 1c left) filled with DMF and $\mathrm{MeOH}$ molecules. The analysis of crystallographic porosity reveals that $66.6 \%$ of the unit cell volume is accessible for solvent. Porosity calculations using Poreblazer 3.0 program $^{29}$ on the crystal structure without solvent molecules results in the geometrical surface area of $2646 \mathrm{~m}^{2} \mathrm{~g}^{-1}$ and pore volume of $1.00 \mathrm{~cm}^{3} \mathrm{~g}^{-1}$.

During the desolvation, however, a transformation from the as made to the cp phase, takes place associated with a distinct color change from green to yellow, and the dense structure without nitrogen accessible porosity is formed (Fig. 1c right).

The crystal structure of the closed structure, DUT-8(Ni) cp, is described here for the first time, as it could only be solved combining single crystal $\mathrm{XRD}$, powder X-ray diffraction and computational simulations. The poor diffraction data, collected on weakly scattering single crystals of DUT-8(Ni) cp phase revealed triclinic unit cell and the structure solution in the
$P 1$ space group provided the coordinates of the Ni atoms (see ESI, $\dagger$ Table S1). This initial model was used for the structure simulation, performed using the modelling and simulation software Material Studio 5.0. ${ }^{30}$ Subsequently, obtained structure was successfully refined against the powder X-ray diffraction data (ESI, $\dagger$ Fig. S1 and S2). The analysis of the crystal structure of cp DUT-8(Ni) shows a changing orientation of the pillaring ligand: dabco molecules, located along [001] direction in as-made phase, are located along [110] in the cp phase. The $\mathrm{Ni}_{2}(2,6-n d c)_{2}$ layers parallel to the (110) plane in the as-made structure are parallel to (101) plane in the cp one.

Analysis of the atoms connectivity in both structures shows that no bond dissociation occurs during the phase transition but, unprecedented, within the paddle-wheel unit the coordination geometry of the $\mathrm{Ni}$ atom changes drastically during the evacuation: thus, in the as-made structure (space group $P 4 / n$ ), the N-Ni-Ni-N atoms of paddle-wheel and dabco molecules are positioned on a common axis forming a linear chain, with $\mathrm{N}-\mathrm{Ni}-\mathrm{O}$ angles of $95.43^{\circ}$ (Fig. 1a). In the cp phase, however, the above mentioned angles are strongly distorted: the $\mathrm{Ni}-\mathrm{Ni}-\mathrm{N}$ angle is $154^{\circ}$ causing the formation of $\mathrm{Ni}-\mathrm{Ni}-\mathrm{N}-\mathrm{N}$ zigzag chains and the values of $\mathrm{N}-\mathrm{Ni}-\mathrm{O}$ angle vary significantly from $82.1^{\circ}$ to $106.8^{\circ}$ (Fig. 1a). The adjacent $\mathrm{O}-\mathrm{Ni}-\mathrm{O}$ angles deform even more dramatic from $89.5^{\circ}$ in the solvent filled form to a wide region of $52.1-128.6^{\circ}$ in the closed phase. However, the distance between two nickel atoms increases only slightly from 2.64 to $2.74 \AA$ in the closed from.

Another soft feature of the structure is the interplanar angles between carboxylates and Ni paddle-wheel that vary from $3.73^{\circ}$ to $47.59^{\circ}$ (Fig. 1b). Thus, in contrast to MIL-53 (MIL - Matériaux de l'Institut Lavoisier), where breathing mechanism is associated purely with changes of the dihedral angle at the carboxylate hinge, structural transformation in DUT-8(Ni) is finally a synergetic effect of hinge deformation (knee-cap dihedral angle deformation) and a unique strong distortion of the square planar paddle-wheel node. Only the combination of cluster and hinge deformation can cause such an enormous change of the pore volume. More intriguing, such a combined mechanism of cluster and hinge deformation is challenging in terms of quantum chemical modelling due to the subtle interplay of van der Walls interactions, bond deformation, and changes of the electronic structure within the cluster. The contraction of the structure leads to drastic changes of the pore window: the channel aperture, measured as a diagonals between oppositely located Ni-paddle-wheels changes from $18.43 \times 18.43 \AA$ in the as-made phase to $23.66 \times 6.95 \AA$ in the cp phase. Such drastic changes of the pore system cause an extensive influence on the porosity. The PLATON provides no accessible void for the guest molecules that is confirmed by Poreblazer 3.0 showing zero surface area and pore volume for the cp structure.

Thus, the structural transformation from the as-made to cp phase results in a very strong contraction of the unit cell volume from $1595 \AA^{3}$ to $647.7 \AA^{3}$ (both recalculated with $Z=1$ ). Consequently the crystallographic density of the framework increases from $0.686 \mathrm{~g} \mathrm{~cm}^{-3}$ to $1.687 \mathrm{~g} \mathrm{~cm}^{-3}$ (if both frameworks without guest molecules are considered). To our knowledge, 
a
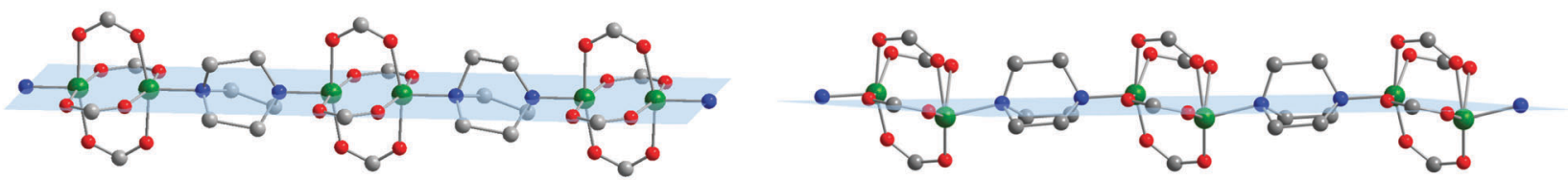

b
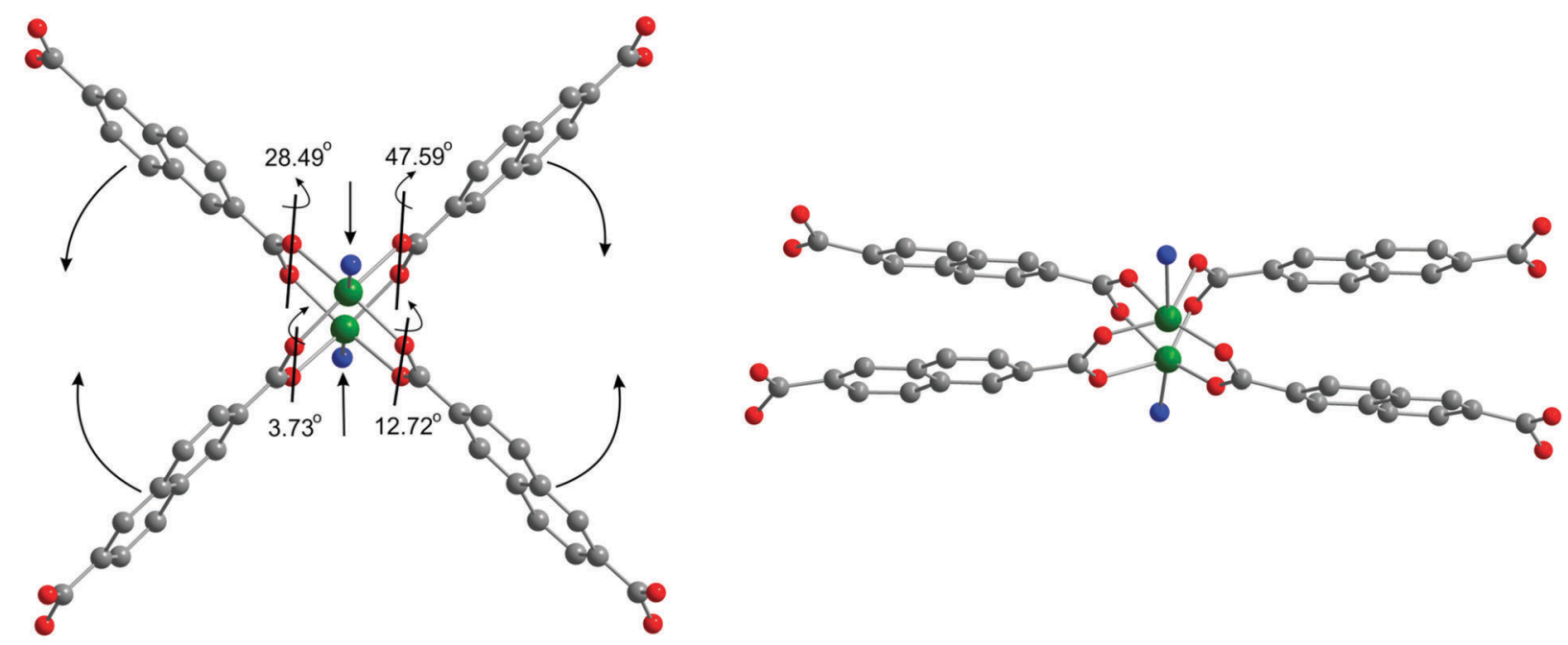

C
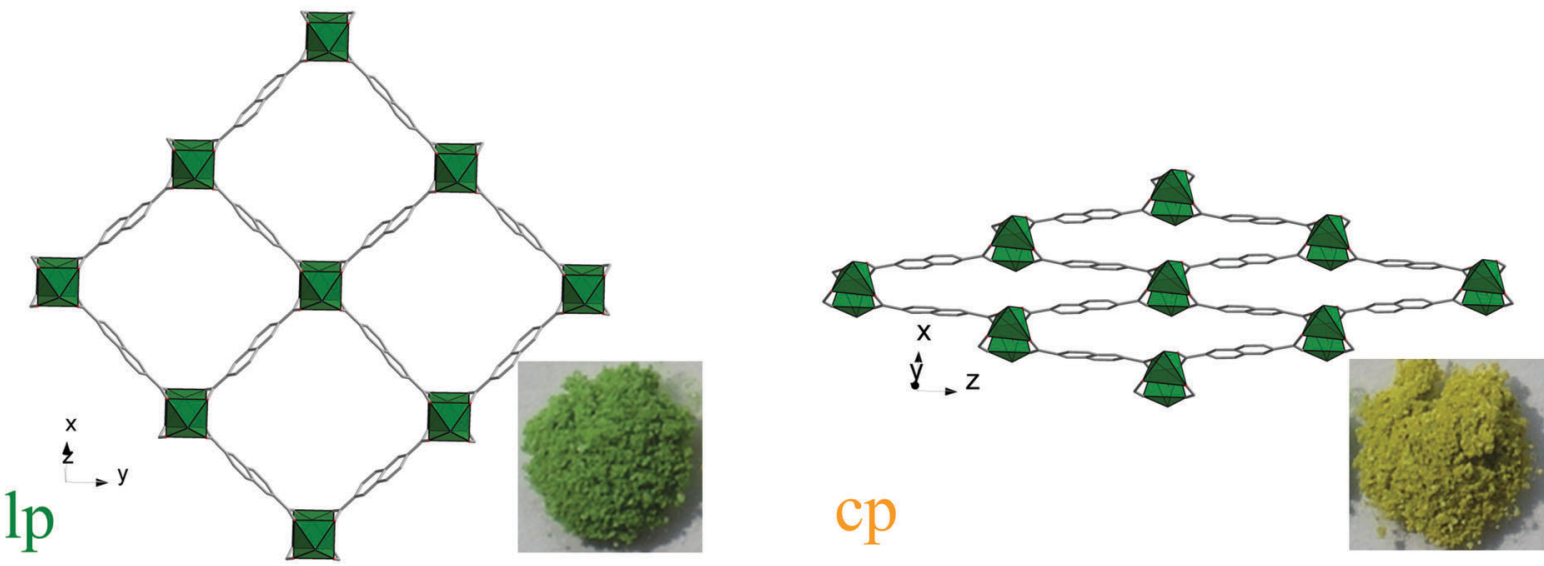

Fig. 1 Structural transformation of DUT-8(Ni): the paddle-wheel chains pillared by dabco molecules (a); SBU environment (b); crystal structure along rectangular channels (c).

this is the largest cell volume change induced by the adsorption of gas molecules in "gate pressure" MOFs ever observed.

For example, the unit cell of MIL-53(Cr) ${ }^{31}$ changes from 1012.6 $\AA^{3}$ in the "narrow pore" phase to $1486.3 \AA^{3}$ in the "large pore" one, corresponding to the $46.8 \%{ }^{31}$ The current record holder, MIL-88D ${ }^{32}$ shows a huge swelling effect during the liquid phase adsorption of solvent molecules, along with an increase of the unit cell volume in the "large pore" phase of 333\% compared to the "closed pore" phase. However, MIL-88 materials exhibit this swelling only in liquid phase adsorption.

In order to explain the gating behavior during the adsorption of gases, parallelized adsorption and powder X-ray diffraction experiments were performed on DUT-8(Ni) at the Helmholtz Center Berlin. ${ }^{18}$ For this purpose, probe molecules with significant differences in polarity and kinetic diameter were chosen as adsorptives.

\section{Adsorption of nitrogen at $77.4 \mathrm{~K}$ monitored in situ by XRD and EXAFS spectroscopy}

Initially, in situ nitrogen physisorption at $77.4 \mathrm{~K}$ was studied, since the ex situ adsorption experiment shows a pronounced hysteresis in the adsorption isotherm (Fig. 2). The powder XRD of starting materials under vacuum shows predominately the presence of cp DUT-8 with a minor impurity of the lp phase (characteristic $(110)$ reflection at $2 \theta=6.8^{\circ}$ ). As described earlier, the activation procedure has a strong influence on the adsorption behavior below the gate opening pressure ${ }^{33}$ and on the phase purity of the compound. The $\mathbf{c p}$ : lp ratio determined from the powder XRD of activated DUT-8(Ni) is $98: 2$.

Interestingly, during the adsorption of small quantities of nitrogen below the "gate pressure", the intensity of lp diffraction peaks decreases, enhancing the ratio of $\mathbf{c p}$ : lp phase from 

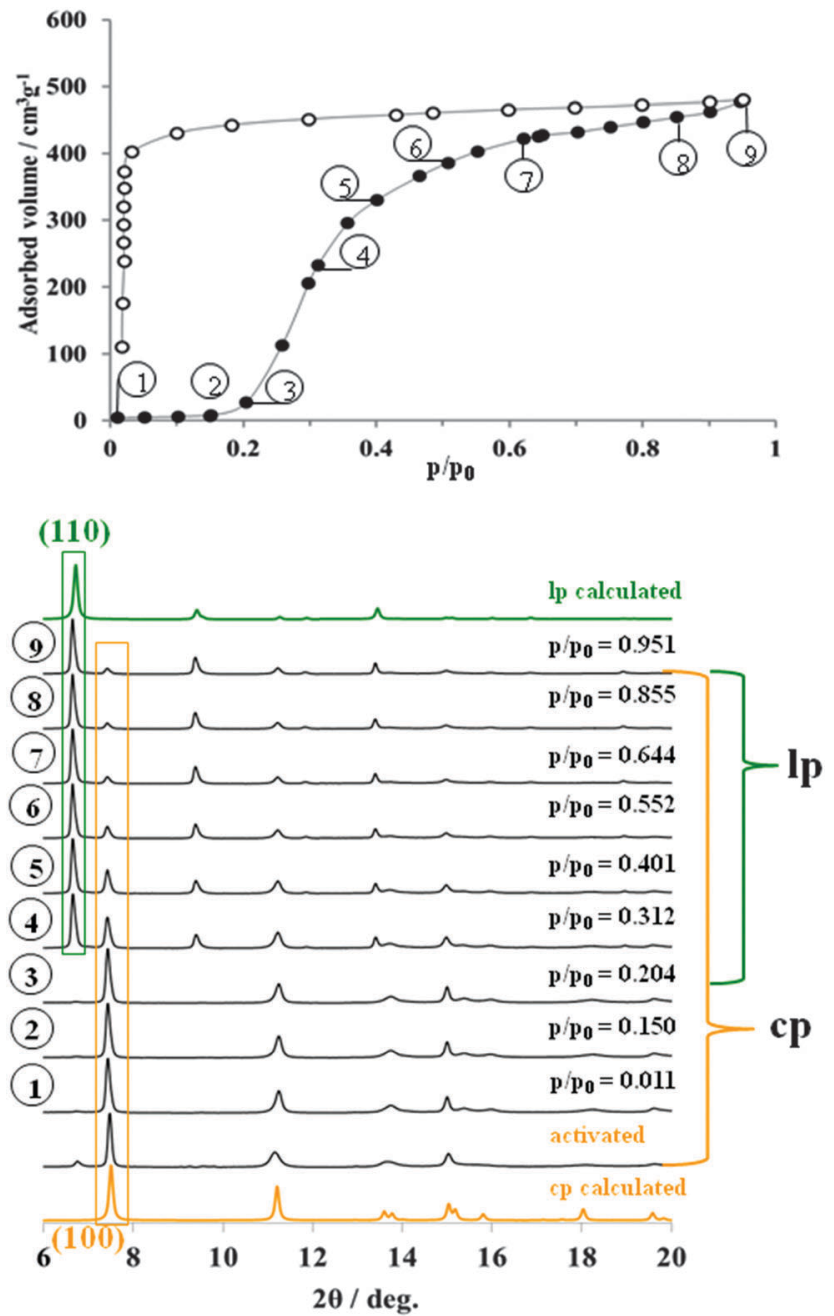

Fig. 2 Adsorption $\mathrm{N}_{2}$ isotherm at $77 \mathrm{~K}$ (top) on DUT-8(Ni) monitored in situ by powder X-ray diffraction (bottom).

$98: 2$ in the evacuated state to $100: 0$ at $p / p_{0}=0.15$ (adsorption point 2, Fig. 2, see also ESI, $\dagger$ Table S4).

Such a phenomenon has been reported earlier and can be interpreted as a sort of re-equilibration in the presence of low gas concentrations indicating a dynamic equilibrium (for example for MIL-53(Cr) during the adsorption of water). ${ }^{31}$ At $p / p_{0}=0.2$, the "gate pressure" is reached accompanied by strong adsorption of nitrogen. The most characteristic (110) reflection of $\mathrm{N}_{2} @ D U T-8(\mathrm{Ni})$ phase appears at $2 \theta=6.8^{\circ}$ in the diffraction patterns collected at relative pressures higher than 0.3. At the same time, the intensity of (100) reflection from the cp phase, continuously decreases with increasing pressure. The ratio of lp: $\mathbf{c p}$ phases derived from the PXRDs phase analysis at the highest measured relative pressure is $59: 41$ (ESI, $\dagger$ Table S4). The PXRD patterns of $\mathrm{N}_{2} @ D U T-8(\mathrm{Ni})$ collected at $p / p_{0}=0.95$ does not exactly match the theoretical pattern of the tetragonal "as made" phase (see ESI, $\dagger$ Fig. S17). The indexing of the PXRD pattern results in a monoclinic cell $\left(P 2_{1} / m\right.$ space group) with cell parameters quite similar to that of the as made phase, but with the monoclinic angle of $94.4^{\circ}$ (Table 1, ESI, $\dagger$ Fig. S6). Interestingly, the unit cell volume of the $\mathrm{N}_{2} @ D U T-8(\mathrm{Ni})$ phase is slightly higher in comparison to the as-made tetragonal phase, making the unit cell expansion during the nitrogen adsorption even more drastic, reaching the level of $254 \%$.

The crystallographic data collected in whole range of relative pressures above the "gate pressure", indicate a step-like transition from cp phase to the $\mathrm{N}_{2} @ D U T-8(\mathrm{Ni})$ phase. Peaks belonging to an intermediate (partially opened) structure are not detected. It is remarkable that the nitrogen uptake of $480 \mathrm{~cm}^{3} \mathrm{~g}^{-1}$, obtained at $p / p_{0}=0.95$ during the in situ experiment (and consistently the corresponding pore volume of $0.743 \mathrm{~cm}^{3} \mathrm{~g}^{-1}$ ) amount to $71.4 \%$ of the theoretical values calculated for "empty" as-made structure (pore volume $1.040 \mathrm{~cm}^{3} \mathrm{~g}^{-1}$ ). In this case, the nitrogen adsorption uptake can to some extent be used as an indicator for the degree of crystal structure transformation.

The changes in the local Ni coordination geometry during the adsorption of nitrogen at $77 \mathrm{~K}$, especially bond lengths in the first coordination shell, were monitored by in situ EXAFS measurements. In the Fig. 3, the fits of the first coordination shell of the Ni scatterers for $\mathbf{c p}$ and $\mathbf{l p}$ phases are shown. For the cp phase the fit shows a good correlation for the Ni-Ni single scattering (SS) path. Thus, according to the crystallographic data the Ni-Ni distance in the cp phase is $2.736 \AA$, whereas EXAFS fit suggests $2.759 \AA$. Because of the low symmetry of the cp structure, as well as a very small spread in $\mathrm{Ni}-\mathrm{O}$ and $\mathrm{Ni}-\mathrm{N}$ distances, it becomes complicated from the physical point of view to fit each SS path separately because of drastically increasing number of parameters. Therefore three Ni-O SS paths with the similar lengths were fitted as one with degeneracy parameter of 3 . The fourth Ni-O SS path with smallest distance as well as Ni-N SS path were fitted separately. Even in this case, the Debye-Waller factors for these paths refine to the negative

Table 1 Unit cell parameters, cell volume, and accessible void volume of DUT-8(Ni) phases studied

\begin{tabular}{|c|c|c|c|c|c|c|c|c|c|c|}
\hline & SG & $a(\AA)$ & $b(\AA)$ & $c(\AA)$ & $\alpha$ (deg) & $\beta$ (deg) & $\gamma(\mathrm{deg})$ & $V\left(\AA^{3}\right)$ & $Z$ & $\begin{array}{l}\text { Void }\left(\AA^{3}\right)(\% \text { of as } \\
\text { made lp phase) }\end{array}$ \\
\hline DUT-8(Ni) cp & $P 1$ & $6.947(1)$ & $8.181(1)$ & $12.172(1)$ & $91.14(1)$ & $103.87(1)$ & $104.55(1)$ & $647.7(1)$ & 1 & $0(0)$ \\
\hline $\mathrm{C}_{2} \mathrm{H}_{6} @ \mathrm{DUT}-8(\mathrm{Ni}) \mathrm{IP1} 184.6 \mathrm{~K}$ & $P 1$ & $9.478(1)$ & $11.066(1)$ & $12.694(1)$ & $101.54(1)$ & $92.06(1)$ & $100.59(1)$ & $1278.5(1)$ & 1 & $729.2(57.0)$ \\
\hline $\mathrm{C}_{2} \mathrm{H}_{4} @ D U T-8(\mathrm{Ni}) \mathrm{IP2} 169.4 \mathrm{~K}$ & $P 1$ & $9.611(1)$ & $11.254(1)$ & $12.556(1)$ & $103.50(1)$ & $94.53(1)$ & $98.32(1)$ & $1296.6(1)$ & 1 & $736.2(56.8)$ \\
\hline DUT-8(Ni) as made $298 \mathrm{~K}$ & $P 4 / n$ & $18.431(1)$ & $18.431(1)$ & $9.391(1)$ & 90 & 90 & 90 & $3190.0(1)$ & 2 & $2124.6(66.6)$ \\
\hline N2@DUT-8(Ni) lp $77 \mathrm{~K}$ & $P 2_{1} / m$ & $18.657(1)$ & $18.736(1)$ & $9.433(1)$ & 90 & $94.42(1)$ & 90 & $3287.5(1)$ & 2 & $2196.0(66.8)$ \\
\hline $\mathrm{CO}_{2} @ \mathrm{DUT}-8(\mathrm{Ni}) \mathrm{lp} 195 \mathrm{~K}$ & $P 2_{1} / m$ & $18.475(1)$ & $18.604(1)$ & $9.431(1)$ & 90 & $95.45(1)$ & 90 & $3226.9(1)$ & 2 & $2156.4(66.8)$ \\
\hline $\mathrm{C}_{4} \mathrm{H}_{10} @ D U T-8(\mathrm{Ni}) \mathrm{lp} 273 \mathrm{~K}$ & $P 2_{1} / m$ & $18.514(1)$ & $18.179(1)$ & $9.409(1)$ & 90 & $95.62(1)$ & 90 & $3151.0(8)$ & 2 & $2080.3(66.0)$ \\
\hline $\mathrm{C}_{2} \mathrm{H}_{4} @ \mathrm{DUT}-8(\mathrm{Ni}) \mathrm{lp} 169.4 \mathrm{~K}$ & $P 2_{1} / m$ & $20.405(1)$ & $16.497(1)$ & $9.347(1)$ & 90 & $94.12(1)$ & 90 & $3138.2(1)$ & 2 & $2070.8(66.0)$ \\
\hline
\end{tabular}



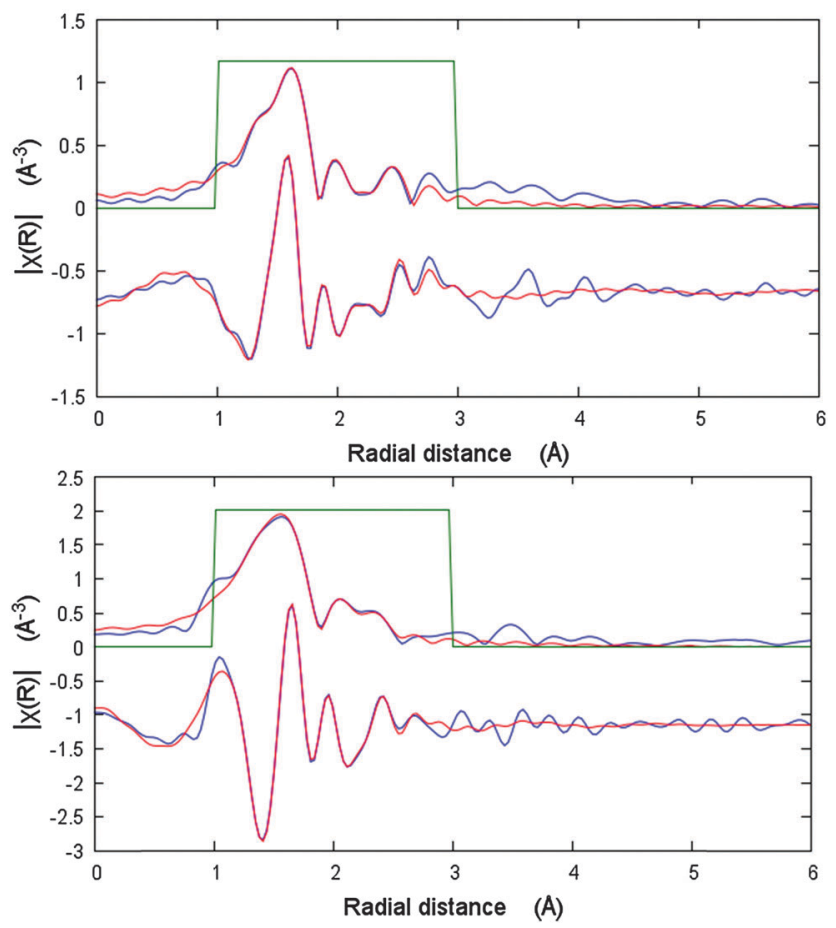

Fig. 3 EXAFS data analysis of DUT-8(Ni) cp (bottom) and $\mathrm{N}_{2}$ (aDUT-8(Ni) Ip (top) phases in $R$-space showing modulus and imaginary part. Blue lines measured data, red line - fit.

values indicating the low reliability of the data. The EXAFS spectrum of the nitrogen filled sample was fitted to the corresponding structural model. Due to the higher symmetry of the Ni-paddle-wheel, only one $\mathrm{Ni}-\mathrm{O}$ path was used with the degeneracy parameter of 4 . Both $\mathrm{Ni}-\mathrm{N}$ and Ni-Ni single scattering paths were fitted in the same manner. In addition, the high symmetry of the SBU as well as high quality of the spectrum allows to involve $\mathrm{Ni}-\mathrm{C}_{\text {carboxylate }}$ path in the fit. As a result, the $\mathrm{Ni}-\mathrm{O}$ SS path was fitted with the distance of $2.017 \AA$ that is $0.158 \AA$ higher in comparison with crystallographic data, reported for as made phase. Opposite, the Ni-Ni, Ni-N and Ni-C SS paths are fitted with the values very close to the crystallographic ones (ESI, $\dagger$ Table S2).

\section{Adsorption of $\mathrm{CO}_{2}$ at $195 \mathrm{~K}$ monitored in situ adsorption by powder XRD}

Compared to nitrogen, the kinetic diameter of carbon dioxide molecules is smaller and the quadrupole moment is higher. This usually results in stronger interaction with the host framework reflected in a higher adsorption enthalpy.

Tuning the adsorption behavior of MOFs for specific gases such as $\mathrm{CO}_{2}$ is essential for the application in separation units. Switchable MOFs show typically guest specific gate opening pressures with significant differences in magnitude for $\mathrm{CO}_{2}$ and $\mathrm{N}_{2} \cdot{ }^{34}$ Such selectivity is also characteristic for DUT-8(Ni). The relative gate opening pressure for $\mathrm{CO}_{2}$ is $c a .0 .4$ at $195 \mathrm{~K}$ and for $\mathrm{N}_{2} c a .0 .2$ (at $77 \mathrm{~K}$ ). In the high pressure region (up to 50 bar at $298 \mathrm{~K}$ ), only $\mathrm{CO}_{2}$ is able to induce the framework opening (gate opening pressure $30 \mathrm{bar}$ ). ${ }^{28}$ The adsorption of
$\mathrm{CO}_{2}$ on DUT-8(Ni) at high pressure was investigated earlier by in situ- ${ }^{13} \mathrm{C}$ NMR spectroscopy. ${ }^{35}$ A certain degree of ordering was observed for all the $\mathrm{CO}_{2}$ molecules adsorbed inside DUT$8(\mathrm{Ni})$ and a tilt angle of $49^{\circ}$ between the symmetry axis and the rotation axis of the molecules adsorbed in the flexible DUT8(Ni) was postulated.

Surprisingly, from the structural point of view, the DUT8(Ni) framework shows at $195 \mathrm{~K}$ carbon dioxide adsorption behavior very similar to that observed during the adsorption of nitrogen at $77 \mathrm{~K}$ (ESI, $\dagger$ Fig. S11). The evacuated sample contains predominantly cp DUT-8 (ESI, $\dagger$ Table S5). After adsorbing of small $\mathrm{CO}_{2}$ amount in the pre-gate region, the (110) peak belonging to lp phase at $2 \theta=6.8^{\circ}$ disappears indicating an increase of the cp phase amount in the bulk material. The (110) reflection of the $\mathrm{CO}_{2} @ D U T-8(\mathrm{Ni})$ phase appears at $p / p_{0}=0.61$ and constantly grows in intensity with increasing $\mathrm{CO}_{2}$ pressure. At the same time the intensity of (100) peak of cp decreases steadily. According to the powder XRD pattern at $p / p_{0}=0.99$, the majority of the sample exist in the large pore $\mathrm{CO}_{2} @ D U T-$ $8(\mathrm{Ni})$ phase (ESI, $\dagger$ Table S5). However both, evaluation of the pore volume at $p / p_{0}=0.99$ as well as quantitative phase analysis of PXRD, suggest the presence of nearly $25 \%$ of closed phase in the sample. The indexing of the $\mathrm{CO}_{2} @ D U T-8(\mathrm{Ni})$ PXRD pattern collected at $p / p_{0}=0.99$ results in a monoclinic cell (space group $P 2_{1} / \mathrm{m}$ ) with monoclinic angle of $95.4^{\circ}$, close to that of $\mathrm{N}_{2} @ D U T-$ $8(\mathrm{Ni})$ phase (Table 1, ESI, $\dagger$ Fig. S7). Thus, the structural changes during the adsorption of $\mathrm{CO}_{2}$ at $195 \mathrm{~K}$ occur in one step and in the similar way as compared to the adsorption of $\mathrm{N}_{2}$ at $77 \mathrm{~K}$.

\section{Adsorption of $\boldsymbol{n}$-butane at $273 \mathrm{~K}$ monitored in situ by powder XRD}

In order to prove the interaction of non-polar hydrophobic adsorptive with the DUT- 8 framework, the pore opening process was studied during the adsorption of $n$-butane at $273 \mathrm{~K}$. At low relative pressures no significant adsorption of $n$-butane was detected (Fig. 4).

The predominating phase in activated sample, according to $\mathrm{X}$-ray diffraction patterns, is the close pore phase. At $p / p_{0}=0.25$, the "gate opening" pressure is reached and DUT-8(Ni) the adsorption starts, accompanied by the corresponding structural changes. Thus, powder XRD patterns, measured in $p / p_{0}$ range from 0.3 to 0.4 indicate the presence of both phases (cp and butane@DUT$8(\mathrm{Ni})$ ) with different ratios (ESI, $\dagger$ Table S3). Even at relative pressure, close to the condensation point, weak (100) reflection belonging to the cp phase is still distinguishable in the powder XRD pattern. As in both previous cases, indexing of the XRD pattern, measured from the $n$-butane filled material results in the monoclinic cell with similar unit cell constants (ESI, $\dagger$ Fig. S8). In order to evaluate the conversion of cp to lp, the pore volume in the point 7 of the isotherm $\left(p / p_{0}=0.95\right)$ was compared with the result of the phase analysis at the same point. The pore volume of $0.71 \mathrm{~cm}^{3} \mathrm{~g}^{-1}$ (67.9\% of the theoretical value) indirectly indicates that not all crystallites undergo the phase transition to the lp phase. As in the case of nitrogen adsorption, the pore volume of the sample strongly corre-

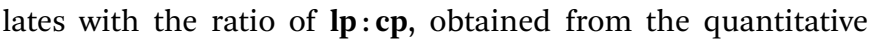



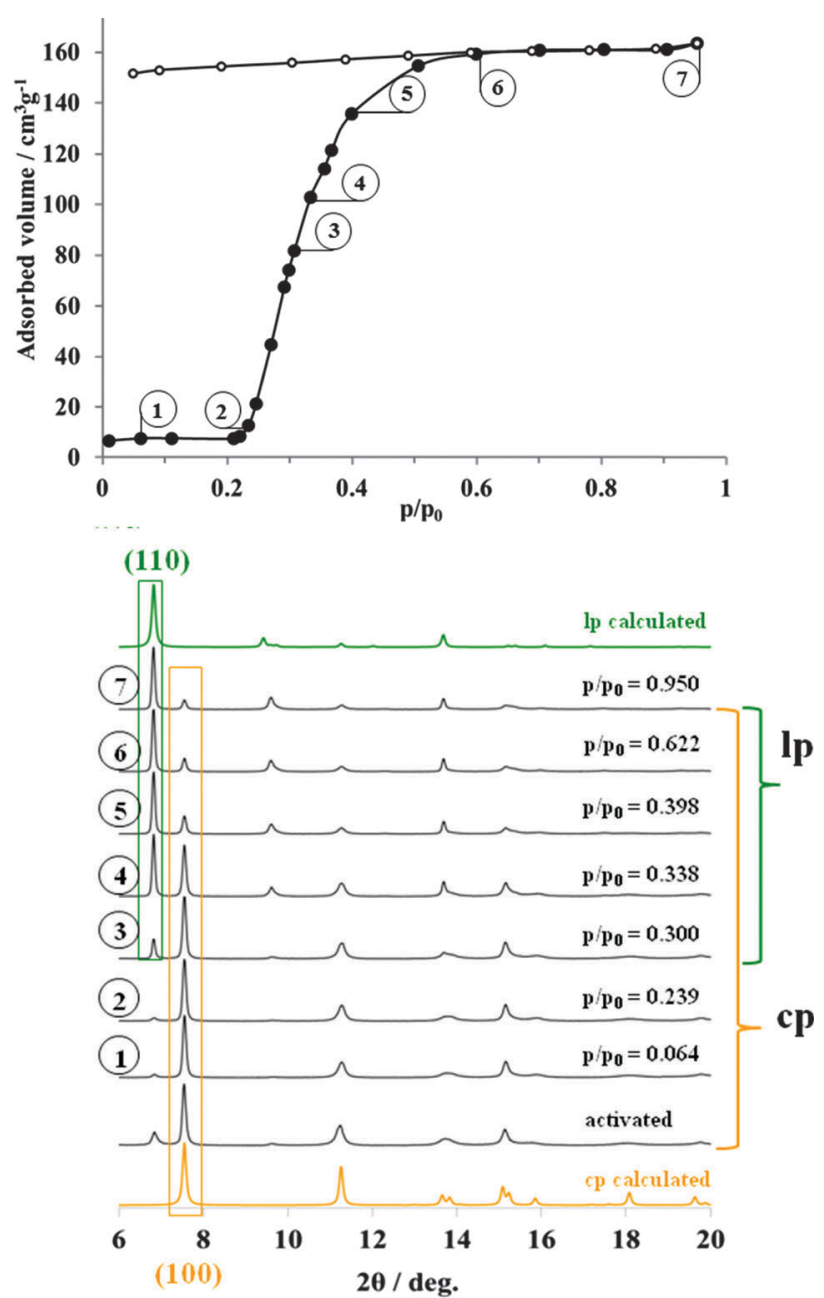

Fig. 4 Adsorption of $n$-butane (272.5 K) on DUT-8(Ni) monitored in situ-XRD.

phase analysis of the powder XRD pattern (lp: $\mathbf{c p}=67: 33$ (ESI, $\uparrow$ Table S3)).

\section{Adsorption of $\mathrm{C}_{2} \mathrm{H}_{6}$ at $185 \mathrm{~K}$ and $\mathrm{C}_{2} \mathrm{H}_{4}$ at $169 \mathrm{~K}$ monitored in situ by powder XRD}

Alkene/alkane separations are of high economic importance for chemical industry. In order to understand selectivity of DUT$8(\mathrm{Ni})$ for ethane $v s$. ethylene, adsorption studies were carried out. The in situ experiments were performed at the saturation temperature for each gas. The changes in the structure start for both gases at nearly the same relative pressure around $p / p_{0}=0.2$. But the network transformation induced by ethane adsorption proceeds along a different structural trajectory in comparison with the probe molecules discussed above. Increasing the pressure, the peaks of the $\mathbf{c p}$ phase decrease in intensity and simultaneously a new reflection at $2 \theta=7.1^{\circ}$ characteristic for the open structure emerges instead of peaks at $2 \theta=6.8^{\circ}$. Obviously, increasing kinetic diameter of the probe molecule leads to changes in the opening mechanism of DUT-8(Ni) and an intermediate phase $\mathrm{C}_{2} \mathrm{H}_{6}$-IP1 forms first.

The formation of intermediate MOF phases and associated multistep adsorption was also recognized previously. ${ }^{36,37}$
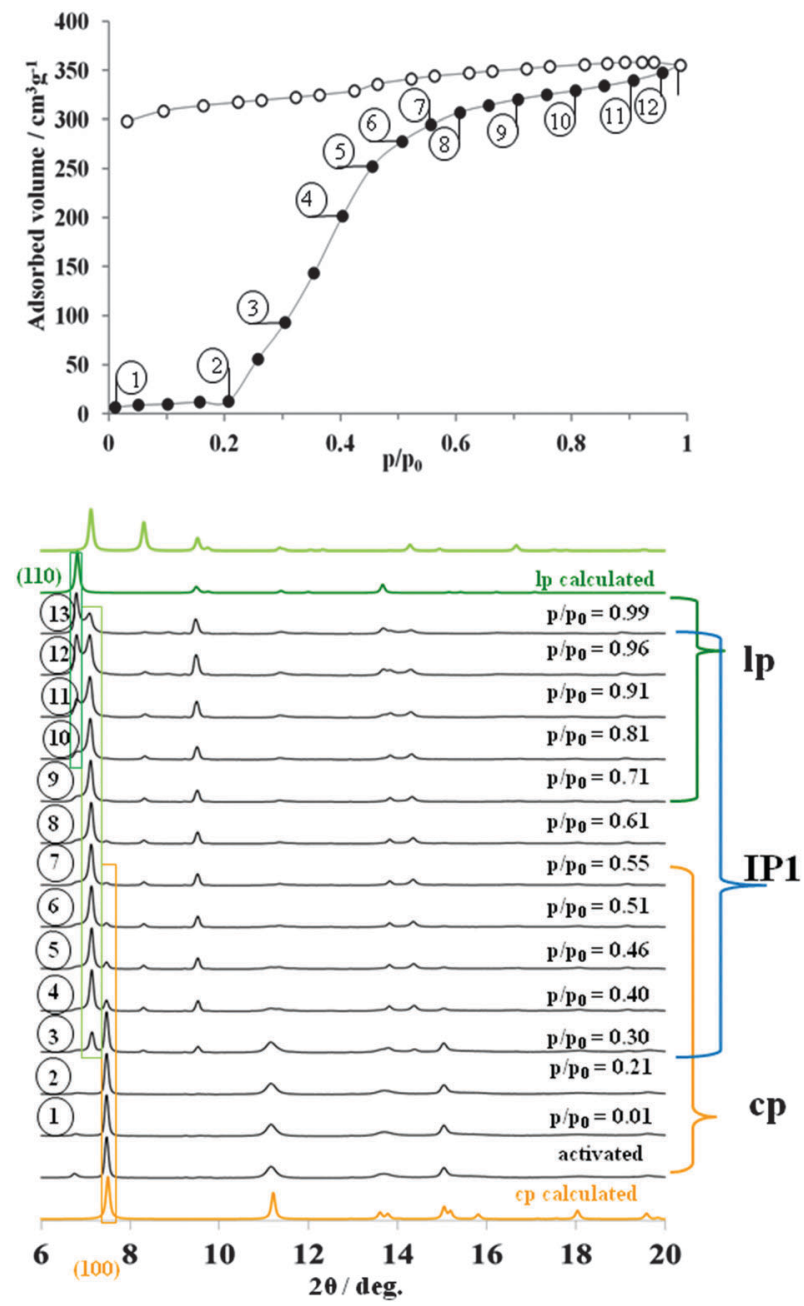

Fig. 5 Adsorption of $\mathrm{C}_{2} \mathrm{H}_{6}(184.6 \mathrm{~K})$ on DUT-8(Ni) monitored in situ by powder XRD.

Indexing of the powder XRD pattern measured at $p / p_{0}=0.61$ (point 8 on the adsorption isotherm in Fig. 5) leads to a triclinic unit cell with commensurate DUT-8(Ni) cell axes and angles (Table 1, ESI, $\uparrow$ Fig. S3). The lattice parameters were used for the geometric optimization of the cp structure using Material Studio 5.0. The resulting model, containing nine ethane molecules per unit cell, was subjected to Rietveld refinement (ESI, $\dagger$ Fig. S3). The number of ethane molecules was estimated from the adsorption isotherm.

The refinement of the crystal structure revealed, that IP1 is based on a distorted paddle-wheel SBU (Fig. 6a). The O-Ni-O angles lie in the narrower range from $79.6(1)^{\circ}$ to $98.8(1)^{\circ}$ in comparison with cp of DUT-8. The Ni-Ni-N angle (characteristic for the linearity of paddle-wheel - dabco axis) evolves from 153.9(1) (bent) in the cp to nearly linear $\left(173.3(1)^{\circ}\right)$ in IP1. It should be mentioned that hinges connected with the paddle-wheel unit play a key role in the pore opening mechanism. The dihedral angles between carboxylate groups and $\mathrm{O}-\mathrm{Ni}-\mathrm{Ni}-\mathrm{O}$ fragments of the paddlewheel cover a range from 121.1 to $152.9^{\circ}$ (Fig. 6a).

During the adsorption, the aperture of the rectangular channels changes from $6.9 \times 23.7 \AA$ in cp phase via $15.1 \times 18.4 \AA$ in the 


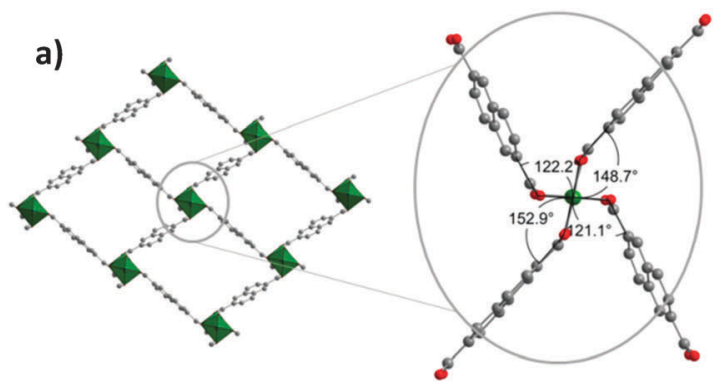

b)

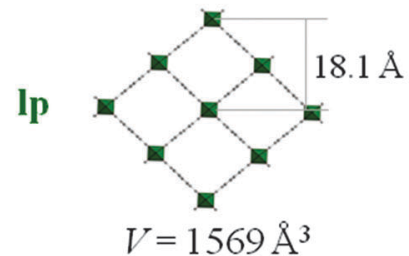

Paddle-wheel distortion

$=1569 \AA^{3}$

IP1

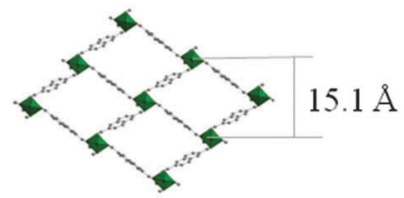

$V=1279 \AA^{3}$

сp

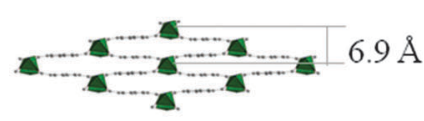

$V=648 \AA^{3}$

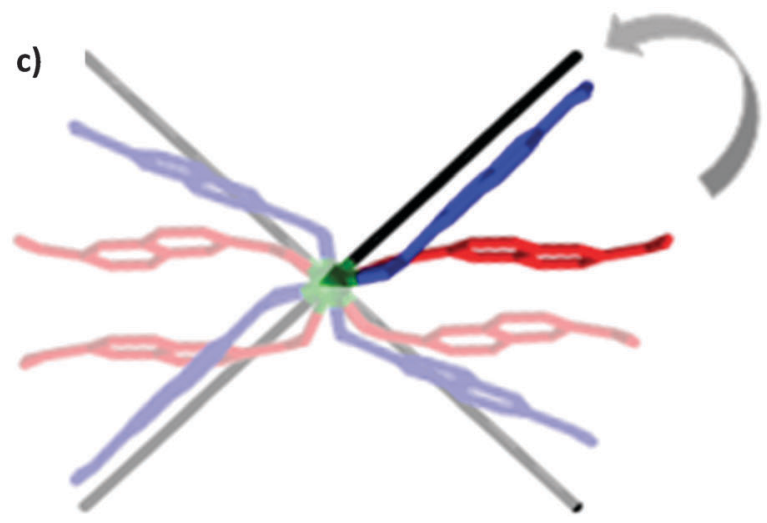

Fig. 6 (a) Coordination geometry of the cluster in IP1 (top). (b) Evolution of the crystal structure and paddle-wheel distortion in DUT-8 cp, IP1 and Ip; (c) Superposition of the paddle-wheels: cp (red), IP1 (blue) and Ip (black)

IP1 up to $18.1 \times 18.6 \AA$ in $\mathrm{C}_{2} \mathrm{H}_{6} @ D U T-8(\mathrm{Ni})$ structure (Fig. 6b and c). According to the powder XRD data, the intermediate phase IP1 is stable at ethane relative pressures from 0.31 to 0.99 . Applying the Poreblazer 3.0 Software on IP1 a geometrical surface area of $1888 \mathrm{~m}^{2} \mathrm{~g}^{-1}$ is estimated, a pore volume of $0.524 \mathrm{~g} \mathrm{~cm}^{-3}$, and a pore limiting diameter of $4.91 \AA$.
The characteristic peak of the lp $\mathrm{C}_{2} \mathrm{H}_{6} @ D U T-8(\mathrm{Ni})$ phase appears in the powder XRD at $p / p_{0}=0.61$ increasing in intensity with increasing relative pressure. It should be mentioned that even at $p / p_{0}=0.99$ the phase analysis of powder XRD patterns shows that only $36.1 \%$ of lp is detected in the sample (ESI, $\dagger$ Table S6), indicating the weak host-guest interactions in the $\mathrm{C}_{2} \mathrm{H}_{6} @ D U T-8(\mathrm{Ni})$ system. The direct comparison of PXRDs of cp, IP1 and lp is given in ESI, $\dagger$ Fig. S12.

Since the ethylene adsorption isotherm at $185 \mathrm{~K}$ is, at a first glance, very similar to the ethane adsorption at $169 \mathrm{~K}$, we assumed the existence of the similar intermediate phase during the gate opening.

However, the analysis of the powder diffraction patterns measured during the adsorption indicates the appearance of a new peak starting at $p / p_{0}=0.202$. At the same time, the positions of the new peaks do not match neither the position of the peaks of the lp phases nor the position of the peaks corresponding to the IP1 phase (ESI, $\dagger$ Fig. S13 and S16).

According to the ethylene adsorption isotherm, an unexpected, novel $\mathrm{C}_{2} \mathrm{H}_{4}$-IP2 (intermediate phase 2) is present in the mixture with cp at $0.20 \leq p / p_{0} \leq 0.25$. In the range of $0.25 \leq p / p_{0} \leq 0.55$, IP2 co-exists in the equilibrium with large $\mathrm{C}_{2} \mathrm{H}_{4}$ @DUT-8(Ni) (ESI, $\dagger$ Fig. S16). Because of the strong overlap of the reflections from all three phases, powder XRDs measured in this range of pressure were not suitable for indexing and further structural analysis. In contrast to all other experiments, complete structural transition from cp to lp phase is achieved at high relative pressures that confirmed by absence of characteristic reflection of cp at $2 \theta=7.6^{\circ}$. The $\mathrm{C}_{2} \mathrm{H}_{4}$ @DUT-8(Ni) lp phase is solved in the $P 2_{1} / m$ space group and shows slightly different cell parameters but very similar to the other guest loaded phases crystal structure (see ESI, $\dagger$ Fig. S5). Interestingly, the reverse structural transition from lp to IP2 proceeds quantitatively and therefore, the powder XRDs, measured during the desorption in the range $0.006 \leq p / p_{0} \leq 0.095$, contain mainly peaks from the IP2. The indexing of one powder XRD in this range resulted in a triclinic unit cell with the lattice parameters and cell volume, very close to that of $\mathrm{C}_{2} \mathrm{H}_{6}$-IP1 (Table 1, ESI, $\dagger$ Fig. S4 and S13). Further desorption of ethylene leads to the formation of $\mathbf{c p}$ phase (ESI, $\dagger$ Fig. S13). Thus, despite the similarities of ethane and ethene adsorption isotherms, subtle structural differences are detected regarding the intermediates formed indicating significant differences in the packing of the small molecules and pore-wall interactions.

\section{Conclusions}

Summarizing, we have shown the importance of combined EXAFS, single crystal and powder XRD measurements parallelized with adsorption methods to reveal the complex structural changes and dynamics of the switchable coordination polymer DUT-8(Ni) in the presence of probe molecules differing in kinetic diameter, polarity and adsorption enthalpy. Especially the strong cluster deformation during $\mathrm{N}_{2}$ and $n$-butane adsorption is unprecedented and has so far only been observed in 
one case. ${ }^{24}$ For nitrogen and carbon dioxide adsorption, DUT$8(\mathrm{Ni})$ shows comparable direct structural transformations from cp to lp phase with a huge expansion of the unit cell volume during the gas adsorption as high as $250 \%$. In the case of $n$-butane, the complete structural transformation to the open phase is hindered. In contrast, ethane and ethene lead to a stepwise adsorption behavior, confirmed by the structural transformations, where intermediate phases could be clearly identified and solved from the powder XRDs, measured at well-defined pressure. Certainly this extent of complex switchability is unique for MOFs. The structural complexity described here for only one MOF (DUT-8(Ni)) demonstrates that through the eye of highly developed in situ-analytical methods there is much more structural chemistry and physics to discover in future. Recognizing soft porous solids as systems in which the structure of the solid cannot be considered without specifying the continuous surrounding phase opens a new way of interpreting structure property relationships.

\section{Acknowledgements}

BMBF (German Federal Ministry of Education and Research) is acknowledged for the financial support (Projects 05K10OD3 and 05K13OD3). We acknowledge the Helmholtz-Zentrum Berlin for provision of travel grants and synchrotron radiation beamtime at beamlines MagS, KMC-2 and MX BL14.2 of BESSY II.

\section{References}

1 O. K. Farha, I. Eryazici, N. C. Jeong, B. G. Hauser, C. E. Wilmer, A. A. Sarjeant, R. Q. Snurr, S. T. Nguyen, A. Ö. Yazaydin and J. T. Hupp, J. Am. Chem. Soc., 2012, 134, 15016-15021.

2 H. Furukawa, N. Ko, Y. B. Go, N. Aratani, S. B. Choi, E. Choi, A. Ö. Yazaydin, R. Q. Snurr, M. O'Keeffe, J. Kim and O. M. Yaghi, Science, 2010, 329, 424-428.

3 M. P. Suh, H. J. Park, T. K. Prasad and D.-W. Lim, Chem. Rev., 2011, 112, 782-835.

4 J.-R. Li, J. Sculley and H.-C. Zhou, Chem. Rev., 2011, 112, 869-932.

5 K. Sumida, D. L. Rogow, J. A. Mason, T. M. McDonald, E. D. Bloch, Z. R. Herm, T.-H. Bae and J. R. Long, Chem. Rev., 2011, 112, 724-781.

6 L. E. Kreno, K. Leong, O. K. Farha, M. Allendorf, R. P. Van Duyne and J. T. Hupp, Chem. Rev., 2011, 112, 1105-1125.

7 A. Schneemann, V. Bon, I. Schwedler, I. Senkovska, S. Kaskel and R. A. Fischer, Chem. Soc. Rev., 2014, 43, 6062-6069.

8 S. Horike, S. Shimomura and S. Kitagawa, Nat. Chem., 2009, 1, 695-704.

9 K. Nakagawa, D. Tanaka, S. Horike, S. Shimomura, M. Higuchi and S. Kitagawa, Chem. Commun., 2010, 46, 4258-4260.

10 R. El Osta, A. Carlin-Sinclair, N. Guillou, R. I. Walton, F. Vermoortele, M. Maes, D. de Vos and F. Millange, Chem. Mater., 2012, 24, 2781-2791.
11 J. Kim, W. Y. Kim and W.-S. Ahn, Fuel, 2012, 102, 574-579.

12 F. Zhang, X. Zou, X. Gao, S. Fan, F. Sun, H. Ren and G. Zhu, Adv. Funct. Mater., 2012, 22, 3583-3590.

13 Q. Chen, Z. Chang, W.-C. Song, H. Song, H.-B. Song, T.-L. $\mathrm{Hu}$ and X.-H. Bu, Angew. Chem., Int. Ed., 2013, 52, 11550-11553.

14 R. Lyndon, K. Konstas, B. P. Ladewig, P. D. Southon, P. C. J. Kepert and M. R. Hill, Angew. Chem., Int. Ed., 2013, 52, 3695-3698.

15 A. C. McKinlay, J. F. Eubank, S. Wuttke, B. Xiao, P. S. Wheatley, P. Bazin, J. C. Lavalley, M. Daturi, A. Vimont, G. De Weireld, P. Horcajada, C. Serre and R. E. Morris, Chem. Mater., 2013, 25, 1592-1599.

16 R. K. Das, A. Aijaz, M. K. Sharma, P. Lama and P. K. Bharadwaj, Chem. - Eur. J., 2012, 18, 6866-6872.

17 S. Bureekaew, H. Sato, R. Matsuda, Y. Kubota, R. Hirose, J. Kim, K. Kato, M. Takata and S. Kitagawa, Angew. Chem., Int. Ed., 2010, 49, 7660-7664.

18 V. Bon, I. Senkovska, D. Wallacher, A. Heerwig, N. Klein, I. Zizak, R. Feyerherm, E. Dudzik and S. Kaskel, Microporous Mesoporous Mater., 2014, 188, 190-195.

19 T. R. Jensen, T. K. Nielsen, Y. Filinchuk, J.-E. Jorgensen, Y. Cerenius, E. M. Gray and C. J. Webb, J. Appl. Crystallogr., 2010, 43, 1456-1463.

20 M. Lange, M. Kobalz, J. Bergmann, D. Lassig, J. Lincke, J. Mollmer, A. Moller, J. Hofmann, H. Krautscheid, R. Staudt and R. Glaser, J. Mater. Chem. A, 2014, 2, 8075-8085.

21 P. L. Llewellyn, P. Horcajada, G. Maurin, T. Devic, N. Rosenbach, S. Bourrelly, C. Serre, D. Vincent, S. LoeraSerna, Y. Filinchuk and G. Férey, J. Am. Chem. Soc., 2009, 131, 13002-13008.

22 S. R. Miller, P. A. Wright, T. Devic, C. Serre, G. R. Férey, P. L. Llewellyn, R. Denoyel, L. Gaberova and Y. Filinchuk, Langmuir, 2009, 25, 3618-3626.

23 P. K. Allan, B. Xiao, S. J. Teat, J. W. Knight and R. E. Morris, J. Am. Chem. Soc., 2010, 132, 3605-3611.

24 J. Seo, C. Bonneau, R. Matsuda, M. Takata and S. Kitagawa, J. Am. Chem. Soc., 2011, 133, 9005-9013.

25 C. Serre, S. Bourrelly, A. Vimont, N. A. Ramsahye, G. Maurin, P. L. Llewellyn, M. Daturi, Y. Filinchuk, O. Leynaud, P. Barnes and G. Ferey, Adv. Mater., 2007, 19, 2246-2251.

26 P. L. Llewellyn, G. Maurin, T. Devic, S. Loera-Serna, N. Rosenbach, C. Serre, S. Bourrelly, P. Horcajada, Y. Filinchuk and G. Ferey, J. Am. Chem. Soc., 2008, 130, 12808-12814.

27 L. Hamon, P. L. Llewellyn, T. Devic, A. Ghoufi, G. Clet, V. Guillerm, G. D. Pirngruber, G. Maurin, C. Serre, G. Driver, W. V. Beek, E. Jolimaître, A. Vimont, M. Daturi and G. R. Férey, J. Am. Chem. Soc., 2009, 131, 17490-17499.

28 N. Klein, C. Herzog, M. Sabo, I. Senkovska, J. Getzschmann, S. Paasch, M. R. Lohe, E. Brunner and S. Kaskel, Phys. Chem. Chem. Phys., 2010, 12, 11778-11784.

29 L. Sarkisov and A. Harrison, Mol. Simul., 2011, 37, 1248-1257.

30 Material Studio, Accelrys Software Inc., San Diego, Release 5.0 edn, 2009. 
31 C. Serre, F. Millange, C. Thouvenot, M. Noguès, G. Marsolier, D. Louër and G. Férey, J. Am. Chem. Soc., 2002, 124, 13519-13526.

32 F. Štěpánek, M. Kubíček, M. Marek, M. Šoóš, P. Rajniak and R. T. Yang, Chem. Eng. Sci., 2000, 55, 431-440.

33 H. C. Hoffmann, B. Assfour, F. Epperlein, N. Klein, S. Paasch, I. Senkovska, S. Kaskel, G. Seifert and E. Brunner, J. Am. Chem. Soc., 2011, 133, 8681-8690.

34 J.-R. Li, Y. Ma, M. C. McCarthy, J. Sculley, J. Yu, H.-K. Jeong, P. B. Balbuena and H.-C. Zhou, Coord. Chem. Rev., 2011, 255, 1791-1823.
35 H. Hoffmann, M. Debowski, P. Müller, S. Paasch, I. Senkovska, S. Kaskel and E. Brunner, Materials, 2012, 5, 2537-2572.

36 F. Salles, G. Maurin, C. Serre, P. L. Llewellyn, C. Knofel, H. J. Choi, Y. Filinchuk, L. Oliviero, A. Vimont, J. R. Long and G. Ferey, J. Am. Chem. Soc., 2010, 132, 13782-13788.

37 V. Bon, I. Senkovska, D. Wallacher, D. M. Többens, I. Zizak, R. Feyerherm, U. Mueller and S. Kaskel, Inorg. Chem., 2014, 53, 1513-1520. 